\title{
Theoretical Modelling Study on the Relationship between Multi-Frequency Microwave Vegetation Index and Vegetation properties (optical depth and single scattering albedo)
}

\author{
Somayeh Talebi ${ }^{1 *}$, Jiancheng Shi ${ }^{1,}$ Tianjie Zhao ${ }^{1,}$
}

\begin{abstract}
State Key Laboratory of Remote Sensing Science, Institute of Remote Sensing and Digital Earth, Chinese Academy of Sciences. Beijing 100101, China; Email: (soma, shijc, zhaotj)@ radi.ac.cn
\end{abstract}

KEY WORDS: Microwave Vegetation Index, Matrix Doubling Model, Optical Depth, Single Scattering Albedo, Soil Moisture, Roughness

\begin{abstract}
:
This paper presents a theoretical study of derivation Microwave Vegetation Indices (MVIs) in different pairs of frequencies using two methods. In the first method calculating MVI in different frequencies based on Matrix Doubling Model (to take in to account multi scattering effects) has been done and analyzed in various soil properties. The second method was based on MVI theoretical basis and its independency to underlying soil surface signals. Comparing the results from two methods with vegetation properties (single scattering albedo and optical depth) indicated partial correlation between MVI from first method and optical depth, and full correlation between MVI from second method and vegetation properties. The second method to derive MVI can be used widely in global microwave vegetation monitoring.
\end{abstract}

\section{INTRODUCTION}

Monitoring vegetation properties by satellites will enhance our understanding of vegetation. Remote sensing-based index is the most popular used tool in vegetation monitoring. Microwave portion of the electromagnetic spectrum $(1 \mathrm{~cm}$ to $1 \mathrm{~m})$ are effective for composition and structure of the surface or volume under observation. Specifically, microwave emissivity varies strongly with surface roughness, polarization, lookangle(England and Johnson 1977) and particularly water content due to liquid water's high permittivity at microwave frequencies (Hunt et al. 2018; Miller 2016; Seo et al. 2010). Investigating on microwave vegetation indices make it clear that they can be influenced by soil emission. This issue can limit application of those products in global vegetation monitoring(Becker and Choudhury 1988; Shi et al. 2008). Shi et al. made a new passive microwave vegetation index (MVI) by AMSRE data from zero-order model, which minimized the surface emission signal and maximized the vegetation signal. The $\tau-\omega$ model represents a zero-order RT solution that links terrain geophysical variables to the observed brightness temperature through soil reflectivity and two vegetation parameters: optical depth and single-scattering albedo (Kerr and Njoku 1990a; Kerr and Njoku 1990b; Mo et al. 1982). As mentioned above, MVI is derived from the zeroth-order radiative transfer solution. The zero-order solution is only applicable at low frequency or for sparse vegetation layer(Ferrazzoli et al. 2002; Kurum et al. 2012). But at high frequencies or dense vegetation, the contribution of scattering within the layer cannot be ignored. This approach will tend to underestimate the total emission. So MVI from zero- order model works well for grasslands, short agricultural crops, and light to moderate vegetation under C-band, otherwise MVIs may not correlate well with vegetation parameters (Chai et al. 2010b). In the present study, MVI in different frequencies based on Matrix doubling model calculated (to take in to account multi scattering effects) and tried to analysis its behavior at different soil moisture and roughness in different densities of corn canopy (a high crop) to understand how it changes. Here, this MVI has been named $\mathrm{MVI}_{\mathrm{B}}^{\mathrm{P}}$. Afterward base on $\mathrm{MVI}_{\mathrm{B}}^{\mathrm{P}}$ limitations we tried to derive MVI-A and MVI-B by a new method. MVI from new method was named $\mathrm{MVI}_{\mathrm{B}}^{\mathrm{T}}$ and $\mathrm{MVI}_{\mathrm{A}}^{\mathrm{T}}$. Then analyzing $\mathrm{MVI}_{\mathrm{B}}^{\mathrm{T}}$ and $\mathrm{MVI}_{\mathrm{A}}^{\mathrm{T}}$ behavior compare to vegetation properties (optical depth and single scattering albedo) has been done. Analyzing these relationships will indicate MVI potential in global vegetation monitoring.

As well as, water cycle observation mission (WCOM) also is designed with new configuration and examine the performance of MVI in multi frequency in the context of this new mission is necessary, so currently the simulation is the only way to do that.

\section{DATA AND METHODOLOGY}

\subsection{Experimental Data}

Detailed vegetation architecture and radiometer data for corn canopy to simulate brightness temperature from Matrix Doubling Model and validating the results were acquired in 2014 in Huailai province China on June and July form 2014.06.22-2014.07.13. Individual plants were defined by sampling randomly selected crop. Measurements for corn were made of leaf width, length and thickness, stalk diameter and length, stalk and leaf moisture, soil and plant temperature, canopy height and stalk length, leaf inclination angle (alpha, beta, and gamma) and density. The vegetation sampling strategy was to choose 5 or 6 moderate ones in growth at different sites around the field-of- view. The terminal value of every parameter was an average of all the measurement. As to soil parameters, a JM624M Platinum resistance thermometer was utilized to obtain the temperature of soil profile, vegetation and environment. Volumetric soil moisture (SMC) was measured at 
different depths also (Chai et al. 2010a). Table 1 is experimental data of corn in Huailai province.

The passive microwave sensor system used in this study was the Truck Mounted Microwave Radiometer (TMMR) (Zhao et al. 2008) which had four-frequency $(6.925 / 10.65 / 18.7 / 36.5 \mathrm{GHz})$ antenna, dual-polarized $(\mathrm{V} / \mathrm{H})$ truck-mounted microwave radiometer designed. The receiver modules by the azimuth and elevation angle of the viewing direction could be changed at a precision of $0.1 \circ$ is fixed on the petitioner. More introductions about TMMR can be found in (Rose and Czekala 2006).

Table1, vegetation parameters for corn

\begin{tabular}{lllll}
\hline \multirow{3}{*}{ Leaf } & Parameters & Unit & Min & Max \\
& Radius & $\mathrm{cm}$ & 1 & 4 \\
& Thickness & $\mathrm{mm}$ & 0.2 & 0.4 \\
& Gravimetric moisture & $\%$ & 0.70 & 0.90 \\
& Angle distribution & degree & 5 & 85 \\
& & & & \\
Stalk & Radius & $\mathrm{cm}$ & 0.2 & 1.2 \\
& Length & $\mathrm{cm}$ & 4 & 140 \\
& Gravimetric Moisture & $\%$ & 0.60 & 0.85 \\
& Angle distribution & degree & 0 & 0 \\
& & & & \\
Layer & Leaf density & $\mathrm{m} 2$ & 52 & 110 \\
& Stalk density & $\mathrm{m} 2$ & 8 & 8 \\
& Layer height & $\mathrm{m}$ & 0. & 2 \\
& & & 11 & \\
\hline
\end{tabular}

In the present study, MVI in different frequencies based on Matrix doubling model calculated. The Matrix Doubling Model has been validated several times using field data,(Eom and Fung 1984; Ferrazzoli et al. 1995; Ferrazzoli et al. 2002; Ferrazzoli et al. 2000) and here we made an additional comparison with TMMR measured brightness temperature. Using the radiometer experiment measurements of the vegetation and soil, simulation from matrix doubling model under different frequencies has been validated. Table 2 is the statistical parameters of comparison TMMR measured brightness temperature and the matrix doubling model simulated for eight days experiment data at vertical and horizontal polarization. $\mathrm{R}$ value of TMMR observed and simulated data shows the correlation coefficient at $\mathrm{C}$ band is more than $\mathrm{X}$ band in both frequencies. Paying attention to $\mathrm{p}$ value indicated the probability of $95 \%$ of the observed relationship is not coincidental.

\begin{tabular}{lclc}
\hline Measured and Simulated BT & $\mathrm{R}^{\wedge} 2$ & RMSE & P-value \\
\hline X band V pol & 0.80 & 3.4 & 0.01 \\
X band H pol & 0.69 & 1.8 & 0.00 \\
C band V pol & 0.84 & 0.8 & 0.00 \\
C band H pol & 0.81 & 3.1 & 0.00 \\
\hline
\end{tabular}

Table2, the statistical parameters of comparison TMMR measured and the matrix doubling simulated brightness temperature for corn.

\subsection{Methodology}

MVI from Multi-Frequency Observations: In the MVI technique the brightness temperature from 0 -order model has been linearly linked to the soil emissivity by two ingredient models (vegetation transmission component as slope and vegetation emission component as intercept). Then to minimize the effect of ground surface emission signal the characteristics of bare surface emission signals at different frequencies are evaluated. The bare soil surface emissivity at two frequencies extremely correlated and the linear function can display this relationship. By eliminating the surface emissivity, brightness temperature observations at a given polarization $\mathrm{P}$ observed with two adjacent frequencies can be describe as a linear function. The intercept and slope of this linear function are the microwave vegetation indices. More information about MVI technique can be found at(Shi et al. 2008).

$$
T_{B p}\left(f_{2}\right)=A_{p}\left(f_{1}, f_{2}\right)+B_{p}\left(f_{1}, f_{2}\right) \cdot T_{B p}\left(f_{1}\right)
$$

$T_{B p}\left(f_{2}\right)$ and $T_{B p}\left(f_{1}\right)$ are the brightness temperature observations at a given polarization $\mathrm{P}$ in a pair of frequency. $B_{p}\left(f_{1}, f_{2}\right)$ and $A_{p}\left(f_{1}, f_{2}\right)$ are MVI-B and MVI-A respectively.

$$
\begin{aligned}
& B_{p}\left(f_{1}, f_{2}\right)=b\left(f_{1}, f_{2}\right) \cdot \frac{V_{t}\left(f_{2}\right)}{V_{t}(f)_{1}} \\
& A_{p}\left(f_{1}, f_{2}\right)=a\left(f_{1}, f_{2}\right) \cdot V_{t}\left(f_{2}\right)+V_{e}\left(f_{2}\right)-B_{p}\left(f_{1}, f_{2}\right) \cdot V_{e}\left(f_{1}\right)
\end{aligned}
$$

$v_{t}$ is vegetation transmission and $v_{e}$ is vegetation emission component at the given frequency; $\mathrm{a}$ and $\mathrm{b}$ are the slope and aspect of linear function of bare surface emissivity at a pair of frequency.

Here MVI is derived from two methods. In plenty and numerous different types of vegetation canopies with different scatter sizes, shapes and orientations, can assume there is no considerable impact of the polarization dependence for vegetation signals (Paloscia et al. 2006; Shi et al. 2008; Van de Griend and Wigneron 2004). The first way to drive MVI here was based on this assumption, by measured brightness temperature at two frequencies:

$$
B\left(f_{1}, f_{2}\right)=\frac{T_{B v}\left(f_{2}\right)-T_{B h}^{t}\left(f_{2}\right)}{T_{B v}\left(f_{1}\right)-T_{B h}^{t}\left(f_{1}\right)}=\frac{\left(\varepsilon_{v}^{s}\left(f_{2}\right)-\varepsilon_{h}^{s}\left(f_{2}\right)\right) \cdot V_{t}\left(f_{2}\right)}{\left(\varepsilon_{v}^{s}\left(f_{1}\right)-\varepsilon_{h}^{s}\left(f_{1}\right)\right) \cdot V_{t}\left(f_{1}\right)}=b\left(f_{1}, f_{2}\right) \cdot \frac{V_{t}\left(f_{2}\right)}{V_{t}(f)_{1}}
$$

The second method to drive MVI was based on (Eq. 1). At this function the slope and intercept of the linear function of brightness temperature simulation in two adjacent frequencies are $B_{p}\left(f_{1}, f_{2}\right)$ and $A_{p}\left(f_{1}, f_{2}\right)$ respectively. Based on theoretical MVI definition $B_{p}\left(f_{1}, f_{2}\right)$ or (MVI-B) and $A_{p}\left(f_{1}, f_{2}\right)$ or (MVIA) are independent of underlying soil surface signals and dependent only on vegetation properties (Shi et al. 2008) So, calculation of MVI-A and MVI-B are possible in different soil parameters, constant canopy properties and constant temperature. We named them $\operatorname{MVI}_{\mathrm{B}}^{\mathrm{T}}$ and $\mathrm{MVI}_{\mathrm{A}}^{\mathrm{T}}$ respectively.

Optical depth and single scattering albedo: Optical depth and single scattering albedo are two vegetation parameters that connect observed brightness temperature from zero order model to geophysical variables in Radiative Transfer Model. Also, based on MVI technique the brightness temperature from 0 order model can be expressed as a linear function of soil emissivity with a slope of $v_{t}$ and intercept of $v_{e}$. Based on $B_{p}\left(f_{1}, f_{2}\right)$ and $A_{p}\left(f_{1}, f_{2}\right)$ definition in Eq. (2) and (3) and their dependency on $v_{t}$ and $v_{e}$ respectively, here this is desirable to understand are $B_{p}\left(f_{1}, f_{2}\right)$ and $A_{p}\left(f_{1}, f_{2}\right)$ as a function of optical depth and single scattering albedo respectively or not? Analyzing MVIs and vegetation properties relationships will indicate MVI potential in global vegetation monitoring. 
To calculate effective optical depth, matrix doubling model is used because of taking in to account multi scattering effects inside the corn canopy. By this method simulated canopy transmissivity of Tor Vergata model is used as a function of the extinction cross section averaged among sub layer scatterers. To calculate effective optical depth by Matrix Doubling Model we used:

$\tau^{*}=-\ln \left(T^{M}\right) \cos \theta$

$\tau^{*}$ is effective optical depth, $T^{M}$ is the transmissivity of corn canopy and $\theta$ is observation angle. Effective albedo by matrix doubling model can be derived as:

$\omega^{*}=1-\frac{e_{i}^{M}}{1-T_{i}^{M}}$

$\omega^{*}$ is effective scattering albedo, $\mathrm{T}^{M}$ is the emissivity simulated by MD model and is observation angle (Ferrazzoli et al. 2002).

\subsection{Result}

First MVI in different frequencies based on Matrix doubling model calculated and tried to analysis its behavior in different soil moisture and roughness by different densities of corn canopy to understand how they change. Figure 1(up) is absolute difference of $\mathrm{MVI}_{\mathrm{B}}^{\mathrm{P}}$ in 0.1 and 0.3 volumetric soil moisture for four types of $\mathrm{MVI}_{\mathrm{B}}^{\mathrm{P}}$. According to the corn graphs, sensitivity to soil moisture was different in different pair of MVI frequencies. As that figure shows MVI-CX, MVI-SL had the least sensitivity to soil moisture, especially MVI-CX(Shi et al. 2008; Zhao et al. 2011). Figure 1(down) is the absolute difference of $\mathrm{MVI}_{\mathrm{B}}^{\mathrm{P}}$ in two roughness. As it clears from the figure, MVI-CX, MVI-SL and MVI-CL were the most in depended and MVI-CS had the most sensitivity to roughness.
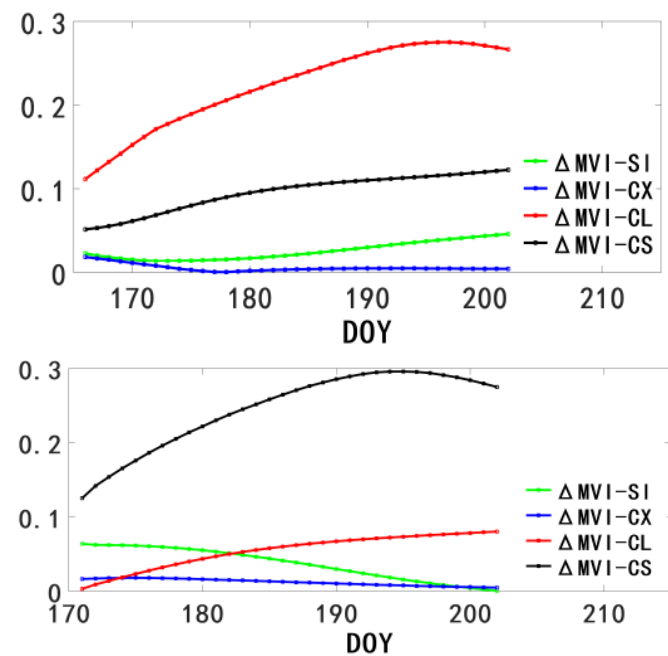

Figure1, Absolut difference of $\mathrm{MVI}_{\mathrm{B}}^{\mathrm{P}}$ for two soil moisture conditions (up), two roughness conditions (down) at 45-degree incident angle.

Although by calculating $\mathrm{MVI}_{\mathrm{B}}^{\mathrm{P}}$ in different frequencies based on Matrix doubling model (to consider multi scattering effects), sensitivity to soil parameters were minimum (Figure1) but still the MVI's behavior compare to soil parameters weren't ideal according to its definition. Yunging Li calculated MVI based on Matrix Doubling Model and tried to explain its's sensitivity to soil moisture and VWC besides other vegetation indexes. She figured out has more stable and smooth trend compare to other microwave vegetation indexes in soil moistures influence but not corresponded completely to theoretical meaning.

The second method to drive MVI was based on the linear function of brightness temperature at two adjacent frequencies (Eq. 1). The slope and intercept of this linear function are and respectively. Figure 2 is $\mathrm{MVI}_{\mathrm{A}}^{\mathrm{T}}$ and $\mathrm{MVI}_{\mathrm{B}}^{\mathrm{T}}$ in four pairs of frequencies. By increasing canopy height $\mathrm{MVI}_{\mathrm{A}}^{\mathrm{T}}$ were increased and $\mathrm{MVI}_{\mathrm{B}}^{\mathrm{T}}$ were decreased in all MVIs(Shi et al. 2008).
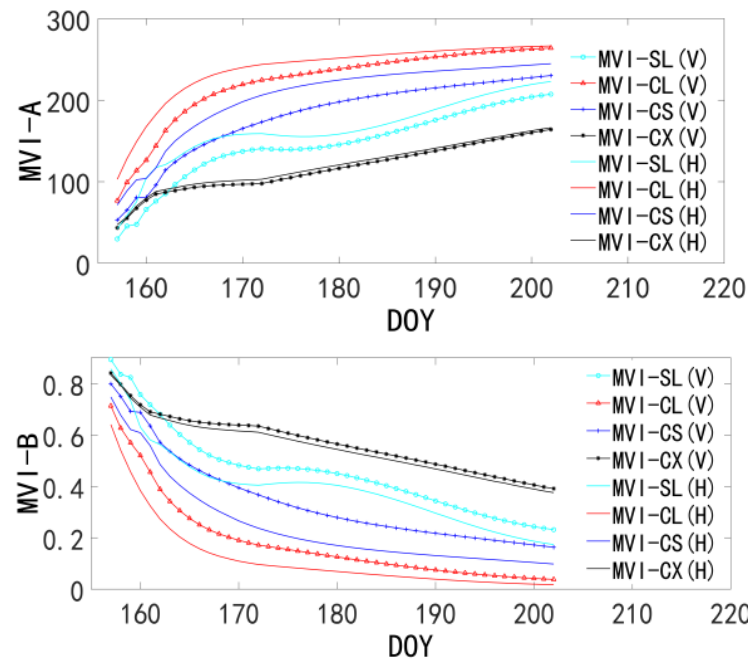

Figure 2, $\mathrm{MVI}_{\mathrm{A}}^{\mathrm{T}}$ (up) and $\mathrm{MVI}_{\mathrm{B}}^{\mathrm{T}}$ (down) in four pairs of frequencies

In this study comparing MVI from two methods to optical depth and single scattering albedo has been done. $\mathrm{MVI}_{\mathrm{B}}^{P}$ and

index in multi frequency (SL, CL, CS and CX) was compared to optical depth at vertical and horizontal polarization and table 3 is the correlation coefficient of comparing them at 45-degree incident angle. The most correlation between $\mathrm{MVI}_{\mathrm{B}}^{P}$ and optical depth in $\mathrm{V}$ and $\mathrm{H}$ polarization (second column) were for MVICX and MVI-SL and they could work well at dense corn (up 2meter). P-values for MVI-CS were more than 0.05 and the correlation of MVI-CL with optical depth in both polarizations were low. The coefficient of determination between optical depth and all four ${ }_{\mathrm{MVI}_{\mathrm{B}}^{\mathrm{T}}}^{\mathrm{T}}$ in both polarizations (third column) generally were more than 90 percent. The important point was higher $\mathrm{R} 2$ values in $\mathrm{MVI}_{\mathrm{B}}^{\mathrm{T}}$ compare to $\mathrm{MVI}_{\mathrm{B}}^{P}$. Paying attention to canopy height shows $\mathrm{MVI}_{\mathrm{B}}^{\mathrm{T}}$ can work well at dense corn (up 2meter). P-values in all cases were less than 0.05 . 


\begin{tabular}{lll}
\hline Comparison type & R2 & R2 \\
\hline MVI-SL, opacity S Band V pol & 0.75 & 0.95 \\
MVI-CL, opacity C Band V pol & 0.13 & 0.95 \\
MVI-CS, opacity C Band V pol & - & 0.98 \\
MVI-CX, opacity C Band V pol & 0.84 & 0.90 \\
MVI-SL, opacity S Band H pol & 0.75 & 0.90 \\
MVI-CL, opacity C Band H pol & 0.15 & 0.89 \\
MVI-CS, opacity C Band H pol & - & 0.92 \\
MVI-CX, opacity C Band H pol & 0.82 & 0.93 \\
\hline Table 3, the statistical parameters of comparison optical depth \\
and four MVI ${ }_{\mathrm{B}}^{P}$ at 45-degree incident angle for V and H
\end{tabular}

Table 4 is coefficient determination of scattering albedo and four $\mathrm{MVI}_{\mathrm{A}}^{\mathrm{T}}$ at 45-degree incident angle in two polarizations for corn from 5-200 centimeter. The correlation coefficients between all $\mathrm{MVI}_{\mathrm{A}}^{\mathrm{T}} \mathrm{s}$ and scattering albedo were high. $\mathrm{R} 2$ values in MVI-Cl and MVI-CS comparison with effective scattering were higher than MVI-SL and MVI-CX. Also, here all four types of $\mathrm{MVI}_{\mathrm{A}}^{\mathrm{T}}$ were acceptable at dense corn. P-value in all cases was less than 0.01 .

The same as what has been done all in above, carried out at 5 , 15,25 and 35 incident angels and the same result were obtained.

\begin{tabular}{ccc}
\hline Comparison type & R2 & P-value \\
\hline MVI-SL, scattering albedo S Band V pol & 0.88 & $3.0596 \mathrm{E}-107$ \\
MVI-CL, scattering albedo C Band V pol & 0.99 & $5.3108 \mathrm{E}-107$ \\
MVI-CS, scattering albedo C Band V pol & 0.99 & $1.11618 \mathrm{E}-98$ \\
MVI-CX, scattering albedo C Band V pol & 0.78 & $1.38042 \mathrm{E}-98$ \\
MVI-SL, scattering albedo S Band H pol & 0.75 & $1.1719 \mathrm{E}-101$ \\
MVI-CL, scattering albedo C Band H pol & 0.98 & $1.085 \mathrm{E}-101$ \\
MVI-CS, scattering albedo C Band H pol & 0.99 & $2.09137 \mathrm{E}-97$ \\
& & \\
MVI-CX, scattering albedo C Band H pol & 0.78 & $2.15016 \mathrm{E}-97$ \\
\hline
\end{tabular}

Table4, the statistical parameters of comparison scattering albedo and four $\mathrm{MVI}_{\mathrm{A}}^{\mathrm{T}}$ at 45-degree incident angle for $\mathrm{V}$ and $\mathrm{H}$ polarization

\section{CONCLUSIONS}

In this paper, we calculated MVI with different assumption by two methods. The characteristic of second method may make it suitable to apply in real satellite images. AMSRE, SMAP and WCOM (in near future) can widely use to apply second method in satellite data. Using second method in different types of vegetation especially for forest and stalk domain canopies can give us significant information in behavior of MVI in different canopy structures and high to study vegetation seasonal changes, phenology and soil moisture retrieval.

\section{REFERENCES}

Becker, F. \& B. J. Choudhury, 1988. Relative sensitivity of normalized difference vegetation index (NDVI) and microwave polarization difference index (MPDI) for vegetation and desertification monitoring. Remote Sensing of Environment 24(2):297-311.

Chai, L., J. Shi, L. Zhang \& T. Jackson, 2010a. Refinement of microwave vegetation indices. In: SPIE Optical Engineering+ Applications, International Society for Optics and Photonics, p 780904-780904-8.

Chai, L., J. Shi, L. Zhang \& L. Jiang, 2010. A parameterized microwave model for short vegetation layer. In: Geoscience and Remote Sensing Symposium (IGARSS), IEEE International, 2010b. Ieee, p 3023 3026.

England, A. \& G. Johnson, 1977. Microwave brightness spectra of layered media. Geophysics 42(3):514-521.

Eom, H. \& A. Fung, 1984. A scatter model for vegetation up to Ku-band. Remote Sensing of Environment 15(3):185200.

Ferrazzoli, P., L. Guerriero, S. Paloscia \& P. Pampaloni, 1995. Modeling $\mathrm{X}$ and $\mathrm{Ka}$ band emission from leafy vegetation. Journal of Electromagnetic Waves and Applications 9(3):393-406.

Ferrazzoli, P., L. Guerriero \& J.-P. Wigneron, 2002. Simulating L-band emission of forests in view of future satellite applications. IEEE Transactions on Geoscience and Remote Sensing 40(12):2700-2708.

Ferrazzoli, P., J.-P. Wigneron, L. Guerriero \& A. Chanzy, 2000. Multifrequency emission of wheat: Modeling and applications. IEEE Transactions on Geoscience and Remote Sensing 38(6):2598-2607.

Hunt, E. R., L. Li, J. M. Friedman, P. W. Gaiser, E. Twarog \& M. H. Cosh, 2018. Incorporation of Stem Water Content into Vegetation Optical Depth for Crops and Woodlands. Remote Sensing 10(2):273.

Kerr, Y. H. \& E. G. Njoku, 1990a. A semiempirical model for interpreting microwave emission from semiarid land surfaces as seen from space. IEEE Transactions on Geoscience and Remote Sensing 28(3):384-393.

Kerr, Y. H. \& E. G. Njoku, 1990b. A semiempirical model for interpreting microwave emission from semiarid land surfaces as seen from space. Geoscience and Remote Sensing, IEEE Transactions on 28(3):384-393.

Kurum, M., P. E. O'Neill, R. H. Lang, A. T. Joseph, M. H. Cosh \& T. J. Jackson, 2012. Effective tree scattering and opacity at L-band. Remote Sensing of Environment 118:1-9.

Miller, B., 2016. Soil Moisture, Vegetation and Surface Roughness Impacts on High Resolution L-Band Microwave Emissivity from Cropped Land during SMAPVEX12. University of Manitoba.

Mo, T., B. Choudhury, T. Schmugge, J. Wang \& T. Jackson, 1982. A model for microwave emission from vegetation - covered fields. Journal of Geophysical Research: Oceans 87(C13):11229-11237.

Paloscia, S., G. Macelloni \& E. Santi, 2006. Soil moisture estimates from AMSR-E brightness temperatures by using a dual-frequency algorithm. IEEE Transactions on Geoscience and Remote Sensing 44(11):31353144.

Rose, T. \& H. Czekala, 2006. Operational manual of RPG$8 \mathrm{CH}-\mathrm{DP} 4$ frequency, dual polarized radiometer. Version.

Seo, D., T. Lakhankar \& R. Khanbilvardi, 2010. Sensitivity analysis of $\mathrm{b}$-factor in microwave emission model for 
The International Archives of the Photogrammetry, Remote Sensing and Spatial Information Sciences, Volume XLII-3, 2018 ISPRS TC III Mid-term Symposium "Developments, Technologies and Applications in Remote Sensing", 7-10 May, Beijing, China

soil moisture retrieval: A case study for SMAP mission. Remote Sensing 2(5):1273-1286.

Shi, J., T. Jackson, J. Tao, J. Du, R. Bindlish, L. Lu \& K. Chen, 2008. Microwave vegetation indices for short vegetation covers from satellite passive microwave sensor AMSR-E. Remote sensing of environment 112(12):4285-4300

Van de Griend, A. A. \& J.-P. Wigneron, 2004. The b-factor as a function of frequency and canopy type at $\mathrm{H}$ polarization. IEEE Transactions on Geoscience and Remote Sensing 42(4):786-794.

Zhao, S., L. Zhang \& Z. Zhang, 2008. Design and test of a new truck-mounted microwave radiometer for remote sensing research. In: Geoscience and Remote Sensing Symposium, IGARSS 2008 IEEE International, 2008. vol 2. IEEE, p II-1192-II-1195.

Zhao, T., L. Zhang, J. Shi \& L. Jiang, 2011. A physically based statistical methodology for surface soil moisture retrieval in the Tibet Plateau using microwave vegetation indices. Journal of Geophysical Research: Atmospheres (1984-2012) 116(D8). 